\title{
KEYNOTE-024: Unlocking a pathway to lung cancer cure?
}

Feature Editor's Note-Non-small cell lung cancer (NSCLC) is responsible for the greatest number of cancerrelated deaths in the United States. The predominant reason for this is that lung cancer is most often diagnosed at advanced stages in which the tumor has spread to lymph nodes or distant organs. Without effective systemic therapy for these cases, cure and extension of life are not possible, and this premise may be extendable to all stages of disease. Nearly one half of all patients diagnosed with NSCLC have distant metastatic (stage IV) disease and when treated with traditional systemic cytotoxic chemotherapy, only $2 \%$ of these individuals are alive 5 years later. Systemic therapy is also a pillar in the treatment of nonmetastatic, advanced-stage (II and III) NSCLC for which 5-year survival remains between 7\% and 36\%. Furthermore, despite early detection and resection for stage I NSCLC, survival at 5 years is only $43 \%$ to $50 \%$, mainly due to the relatively high frequency of distant recurrence for which systemic therapy is essential. Thus, NSCLC is a malignancy for which effective systemic therapy is requisite for improving progression-free and overall survival in potentially every stage of its disease, and based on our current outcomes, the state of systemic therapy is fundamentally insufficient.

Enter the immunotherapeutic revolution. It has only been within the past several years that immune checkpoint inhibitors have revolutionized the treatment of patients with advanced NSCLC, providing improved systemic therapy and durable clinical benefit in many cases. In the accompanying Feature Editorial, Rusch and colleagues review the results of the landmark clinical trials on checkpoint blockade for unresectable stage III and IV NSCL and the basis for the recent US Food and Drug Administration approval of the PD-1 inhibitor, pembrolizumab (Keytruda), as first-line therapy for metastatic NSCLC. The discussion is extended to the promise of immune checkpoint blockade to favorably impact patients with surgically resectable stage I and II

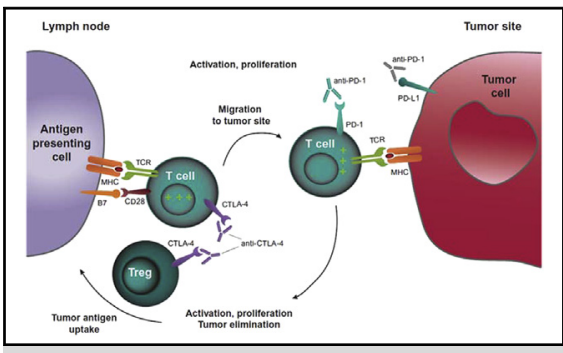

Schematic representation of the effects of CTLA-4 and PD-1 pathway blockade. Used with permission.

Central Message

A new class of drugs, T-cell checkpoint inhibitors, which augment antitumor immune response, have substantially improved outcomes in advanced lung cancer and are now being tested in resectable lung cancer.

See Editorial Commentary page 1781.

NSCLC. Taken together with the rapid pace of this field, we can expect to see a central role for checkpoint blockade in the treatment of all stages of NSCLC, and it can be predicted that immune checkpoint inhibitors will improve the disparaging survival statistics that we have become accustomed to referencing.

\section{Bryan M. Burt, MD}

For 30 years, the standard systemic treatment for metastatic and locally advanced non-small cell lung cancer (NSCLC) was platinum-based chemotherapy. This began to change a decade ago with the discovery that some NSCLCs, particularly adenocarcinomas, harbor specific "driver" mutations that confer exquisite sensitivity to orally administered tyrosine kinase inhibitors (TKIs). After sensitizing mutations in epidermal growth factor receptor $(E G F R)$ were initially reported in 2004, other "actionable" genetic abnormalities that predicted response to various TKI were rapidly identified. ${ }^{2-4}$ Approximately one third of lung adenocarcinomas are now found to contain such actionable genetic abnormalities. As a result, metastatic lung cancer is no longer treated as a single homogenous malignancy but understood to be a heterogenous disease for which molecular profiling is standard and can help identify optimal therapies. ${ }^{5}$ In short, recognition of the distinct molecular underpinning of individual tumors originated the era of personalized medicine for lung cancer, revolutionizing treatment options and substantially improving overall survival (OS) for selected patients. However, resistance to 
Lymph node

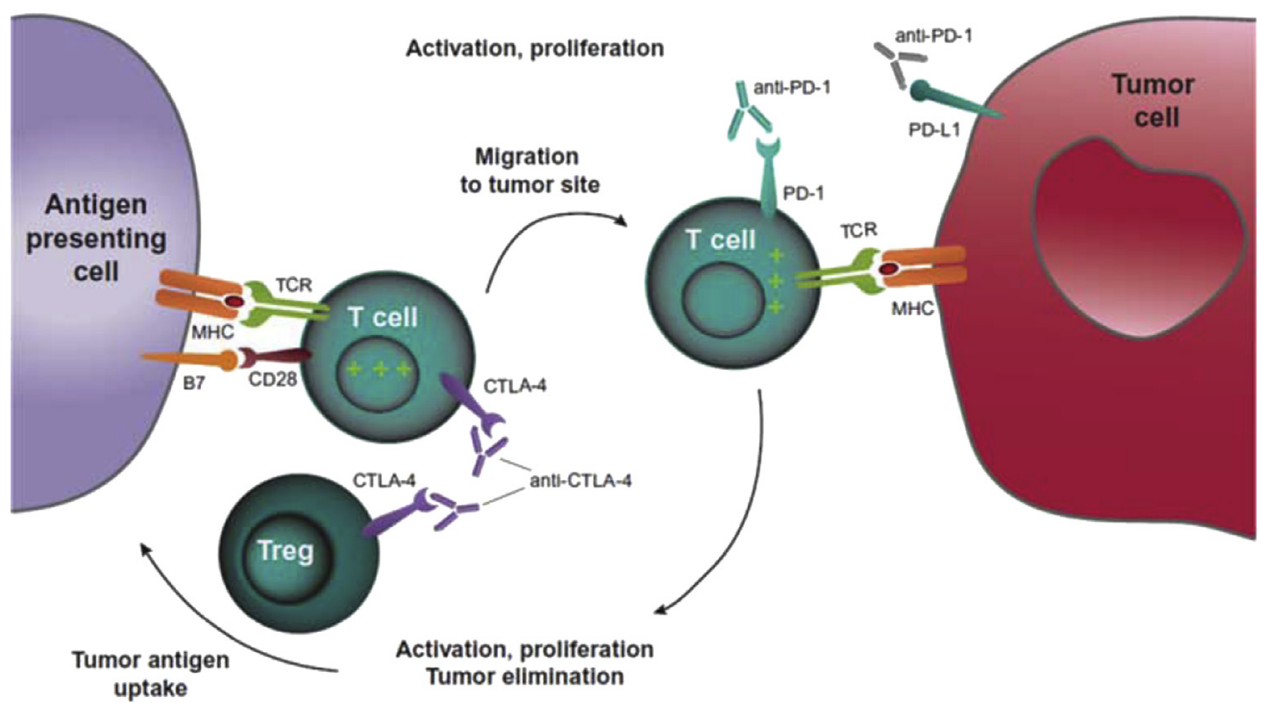

FIGURE 1. Schematic representation of the effects of CTLA-4 and PD-1 pathway blockade. Used with permission. ${ }^{1}$

TKI "targeted therapies" occurs in most patients after 1 to 2 years because of the development of secondary mutations. New TKIs have been developed to address this problem, ${ }^{6}$ but although such drugs substantially extend life expectancy, they remain noncurative.

Following the molecular revolution in lung cancer, the last 5 years have been defined by the immune revolution. In 2010, the first of a new class of monoclonal antibodies, ipilimumab, designed to enhance tumor immunity and thereby address the fundamental problem of cancers escaping immune surveillance, was reported to improve OS in patients with metastatic melanoma. ${ }^{7}$ Ipilimumab, a fully human, IgG1 monoclonal antibody, blocks cytotoxic T-lymphocyte-associated antigen 4 (CTLA-4). Subsequently, other so-called T-cell "checkpoint inhibitors," such as nivolumab or pembrolizumab, that play a complementary role in regulating adaptive immunotherapy (Figure 1) by blocking the programmed death 1 receptor (PD-1) or its ligand (PD-L1) were developed. ${ }^{1}$ In advanced melanoma, the combination of nivolumab and ipilimumab yielded a greater response rate but at the cost of greater toxicity. ${ }^{8}$ Approximately $25 \%$ of patients with advanced NSCLC have a high level of PD-L1 expression and were found in early clinical trials to respond to nivolumab and pembrolizumab, providing a rationale to compare them with standard cisplatin-based chemotherapy.,

KEYNOTE-024 ${ }^{11}$ was a phase 3 trial in which 305 patients who had untreated, advanced NSCLC with high PD-L1 expression were randomized to receive either pembrolizumab (anti-PD-1 therapy) or the investigator's choice of platinum doublet chemotherapy. Patients with sensitizing EGFR mutations or translocation of the anaplastic lymphoma kinase $(A L K)$ gene were excluded.
Crossover therapy was permitted on both arms of the trial for patients who developed progressive disease after firstline therapy.

The trial was unequivocally positive, meeting both its primary and secondary endpoints. Patients treated with pembrolizumab had significantly better OS, progression-free survival, (PFS), as well as overall response rate compared with chemotherapy. An updated analysis of this trial, presented at the 2017 meeting of the American Society of Clinical Oncology, showed that fewer patients randomized to first-line pembrolizumab received additional therapy because of its efficacy in the first-line setting and that the time to progression on second-line therapy was also improved in those initially treated with pembrolizumab. ${ }^{12}$

As a result of KEYNOTE-024, pembrolizumab was approved by the US Food and Drug Administration as first-line therapy for patients with NSCLC with high PDL1 expression. In addition, PD-L1 immunohistochemistry is now routinely recommended in all patients with a new diagnosis of advanced NSCLC, as it informs the potential use of pembrolizumab in the first line. Of note, smaller studies have even shown that pembrolizumab has singleagent activity in patients with brain metastases. ${ }^{13}$

T-cell checkpoint inhibitors have radically altered the approach to systemic therapy in lung cancer and many other solid tumors and have spawned an explosive number of clinical trials. As of August 2017, the clinicaltrials.gov Web site listed more than 250 active and 50 planned checkpoint inhibitor trials in lung cancer alone. ${ }^{14}$ These studies address many gaps in our knowledge about these agents, including who are the best patients to treat with immunotherapies and whether efficacy can be augmented through combination with cytotoxic chemotherapy, radiation, oncolytic viral 
therapy, or other checkpoint modulators (eg, CTLA-4). ${ }^{15}$ Rarely, dramatic responses to checkpoint inhibitor therapy occur to the extent that patients with advanced NSCLC have undergone potentially curative resection of the residual tumor sites. Continued disease remission may be seen after discontinuation of checkpoint inhibitors, and the optimal duration of treatment remains undefined. ${ }^{16}$

After 30 years of little progress in systemic therapy for lung cancer, the level of excitement in the oncologic community about this new therapeutic approach is understandable. However, the current progress represents a beginning rather than an end, and there is much work left to be done. For example, unlike the "targeted therapies" to which response can be predicted based on specific molecular abnormalities, the factors governing response to T-cell checkpoint inhibitors are still poorly understood. In KEYNOTE-024, PD-L1 expression (as assessed by immunohistochemistry) on at least $50 \%$ of tumor cells was an eligibility criterion because preceding trials suggested that this threshold was associated with a greater response rate and improved OS. ${ }^{11}$ PD-L1 expression, however, is not entirely sensitive or specific. More than one half of tumors with high PD-L1 expression do not respond to pembrolizumab, and, conversely, responses can occur among patients who are PD-L1 negative. In addition, PD-L1 immunostaining is only reliable in larger amounts of tissue, ie, on histology but not cytology specimens. Additional biomarkers are needed. Whole-exome sequencing of NSCLC has shown that greater nonsynonymous mutation burden is associated with objective response, durable clinical benefit, and PFS in patients treated with pembrolizumab. ${ }^{17}$ Sensitivity to immune checkpoint blockade in lung cancer and other solid tumors has also been correlated with DNA mismatch repair deficiency, which in turn is associated with a very large number of mutation-associated neoantigens that can be recognized by the immune system. ${ }^{18}$ In contrast, NSCLCs that have driver mutations (eg, EGFR mutations) that confer sensitivity to targeted therapies and that presumably have a low mutation burden have a low likelihood of responding to checkpoint inhibitors. ${ }^{19}$ Despite these initial insights, a better understanding of the mechanisms underlying response is needed to refine patient selection for T-cell checkpoint inhibitor therapy. Correlative studies to identify the factors determining response are critical aspects of ongoing clinical trials.

In light of the success of T-cell checkpoint inhibitor therapy in advanced lung cancer, these drugs are now being tested in the treatment of locally advanced NSCLC. Recently, a phase 3 study of adjuvant durvalumab (antiPD-L1) in patients with stage III NSCLC without progression immediately after concurrent chemoradiation has demonstrated a greater than 11-month improvement in PFS and is expected to become a new standard of care for patients in this setting. ${ }^{20}$
With respect to resectable NSCLC, the initial results of a small, 2-institution trial using 2 doses of neoadjuvant nivolumab over 4 weeks in this patient population were reported at the 2017 American Society of Clinical Oncology meeting. Of 21 patients treated with operative intent, 20 underwent resection with no delays in the planned operations or unusual perioperative toxicities. A major pathologic response (defined as $\leq 10 \%$ residual viable tumor at the time of resection) was achieved in 9 of $21(43 \%)$ patients. This is far greater than has been seen with neoadjuvant chemotherapy. ${ }^{21}$ Pretreatment tumor exome sequencing showed a correlation between pathologic response and both tumor mutation and predicted neoantigen loads. ${ }^{22}$ These encouraging results confirm the rationale for moving forward with multiple ongoing clinical trials testing induction and adjuvant checkpoint inhibitor therapy in patients with locally advanced, resectable NSCLC. Neoadjuvant trials are particularly important because they allow correlative studies to be performed on pre- and postinduction treatment tumor samples, which should elucidate determinants of response to checkpoint inhibitors.

It is important for thoracic surgeons to become conversant with T-cell checkpoint inhibitor therapy because it will likely transform the treatment of locally advanced NSCLC just as it has for metastatic lung cancer. In the same way that surgeons had to learn the pitfalls of operating after induction chemo- and chemoradiotherapy 30 years ago, checkpoint inhibitors pose their own set of challenges. Although the overall severity of adverse events with these antibodies tends to be less than with platinum-based chemotherapy, checkpoint inhibitor therapy has unique toxicities. It is contraindicated in patients who have underlying autoimmune diseases (eg, inflammatory bowel disease) and has been associated with a low frequency but wide range of immune-related complications, including colitis, pneumonitis, hyper- or hypothyroidism, adrenal insufficiency, nephritis, various cardiotoxicities, and uveitis. ${ }^{23,24}$ These toxicities appear to be less common with the anti-PD-1/ PD-L1 than with the CTLA-4 antibodies. Specifically, the risk of pneumonitis with agents such as nivolumab and pembrolizumab appears to be in the $5 \%$ range $^{25}$ but can require high-dose steroid therapy and has rarely resulted in death. Whether a patient who had pneumonitis during induction therapy needs pulse-dose steroids to prevent a flare of this during the perioperative period is unknown. Defining the frequency and severity of these immune-related adverse events and their impact on surgical management is a critical aspect of the current generation of induction therapy trials.

In addition, there may be a discrepancy between radiologic and pathologic response that can confuse planning or executing surgical resection. "Pseudoprogression," defined as radiographic tumor progression without clinical deterioration from apparent tumor growth and without pathologic progression, has been reported in up to $15 \%$ of patients with 
melanoma but is uncommon in lung cancer. ${ }^{1}$ Nevertheless, early experience with resection of NSCLC after checkpoint inhibitor therapy confirms a sometimes-unpredictable discrepancy between radiologic and gross surgical findings versus pathologic response. Specifically, patients may have a sizeable residual mass that on final pathology shows no viable tumor, only residual fibrosis. There may also be intense fibrosis or inflammatory response in lymph nodes that makes hilar dissection difficult. Although this does not seem to occur frequently, it important for surgeons to understand that radiologic and gross appearances can be deceiving and that some resections may prove technically challenging. Again, the current generation of neoadjuvant trials will be important in defining these surgical issues further.

T-cell checkpoint inhibitor therapy is unquestionably revolutionizing the landscape of lung cancer treatment. It will alter the approach to multimodality care in this disease, may lead thoracic surgeons to operate on some patients who would not previously have survived their initial metastatic disease, and will present some new challenges in surgical management. This is now a field that is evolving rapidly, with new clinical trial data and biologic discoveries nearly every day. Stay tuned.

\section{Conflict of Interest Statement}

Dr Rusch: clinical trial funded by Genentech, unpaid member of Mesothelioma Scientific Advisory Board for BMS; Dr Chaft: consultancies with AZ, BMS, Merck, and Genentech; and Dr Hellmann: research grant from BMS, personal fees for consulting from Genentech, Merck, BMS, AZ, Novartis, Janssen, Mirati, and Shattuck labs.

\section{References}

1. Buchbinder EI, Desai A. CTLA-4 and PD-1 pathways. Similarities, differences, and implications of their inhibition. Am J Clin Oncol. 2016;39:98-106.

2. Paez JG, Jänne PA, Lee JC, Tracy S, Greulich H, Gabriel S, et al. EGFR mutations in lung cancer: correlation with clinical response to gefitinib therapy. Science. 2004;304:1497-500.

3. Kwak EL, Bang Y-J, Camidge DR, Shaw AT, Solomon B, Maki RG, et al. Anaplastic lymphoma kinase inhibition in non-small cell lung cancer. $N$ Engl J Med. 2010;363:1693-703.

4. Bergethon K, Shaw AT, Ou S-HI, Katayama R, Lovly CM, McDonald NT, et al ROS 1 rearrangements define a unique molecular class of lung cancers. J Clin Oncol. 2012;30:863-70.

5. Ettinger DS, Wood DE, Aisner DL, Akerley W, Bauman J, Chirieac LR, et al. Non-small cell lung cancer, Version 5.2017. J Natl Compr Canc Netw. 2017; 15:504-35.

6. Mok TS, Wu Y-L, Ahn M-J, Garassino MC, Kim HR, Ramalingam SS, et al. Osimertinib or platinum-pemetrexed in EGFR T790M-positive lung cancer. $N$ Engl J Med. 2017;376:629-40
7. Hodi FS, O'Day SJ, McDermott DF, Weber RW, Sosman JA, Haanen JB, et al. Improved survival with ipilimumab in patients with metastatic melanoma. $N$ Engl J Med. 2010;363:711-23.

8. Wolchock JD, Kluger H, Callahan MK, Postow MA, Rizvi NA, Lesokhin AM, et al. Nivolumab plus ipilimumab in advanced melanoma. $N$ Engl J Med. 2013;369:122-33

9. Garon EB, Rizvi NA, Hui R, Leighl N, Balmanoukian AS, Eder JP, et al. Pembrolizumab for the treatment of non-small cell lung cancer. $N$ Engl J Med. 2015:372:2018-28.

10. Borghaei H, Paz-Ares L, Horn L, Spigel DR, Steins M, Ready NE, et al. Nivolumab versus docetaxel in advanced nonsquamous non-small-cell lung cancer. $N$ Engl J Med. 2015;373:1627-39.

11. Reck M, Rodriguez-Abreu D, Robinson AG, Hui R, Csöszi T, Fülöp A, et al. Pembrolizumab versus chemotherapy for PD-L1-positive non-small cell lung cancer. N Engl J Med. 2016;375:1823-33.

12. Brahmer JR, Rodriguez-Abreu D, Robinson AG, Hui R, Csöszi T, Fülöp A, et al. Progression after the next line of therapy (PFS2) and updated OS among patients with advanced NSCLC and PD-L1 TPS $\geq 50 \%$ enrolled in KEYNOTE-024. $J$ Clin Oncol. 2017;35(suppl). abstr 9000.

13. Goldberg SB, Gettinger SN, Mahajan A, Chiang AC, Herbst RS, Sznol M, et al. Pembrolizumab for patients with melanoma or non-small cell lung cancer and untreated brain metastases: early analysis of a non-randomised, open-label, phase 2 trial. Lancet Oncol. 2016;17:976-83.

14. United States National Institutes of Health. Clinical Trials.gov. Available at: https://clinicaltrials.gov/. Accessed September 1, 2017.

15. Hellmann MD, Rizvi NA, Goldman JW, Gettinger SN, Borghaei H, Brahmer JR, et al. Nivolumab plus ipilimumab as first-line treatment for advanced non-small cell lung cancer (CheckMate 012): results of an open-label, phase 1, multicohort study. Lancet Oncol. 2017;18:31-41.

16. Chaft JE, Hellmann MD, Velez MJ, Travis WD, Rusch VW. Initial experience with lung cancer resection after treatment with T-cell checkpoint inhibitors. Ann Thorac Surg. 2017;104:e217-8.

17. Rizvi NA, Hellmann MD, Snyder A, Kvistborg P, Makarov V, Havel JJ, et al. Mutational landscape determined sensitivity to PD-1 blockage in non-small cell lung cancer. Science. 2015;348:124-8.

18. Le DT, Durham JN, Smith KN, Wang H, Bartlett BR, Aulakh LK, et al. Mismatch repair deficiency predicts response of solid tumors to PD-1 blockade. Science. 2017:357:409-13.

19. Lee CK, Man J, Lord S, Links M, Gebski V, Mok T, et al. Checkpoint inhibitors in metastatic EGFR-mutated non-small cell lung cancer-a meta-analysis. J Thorac Oncol. 2017;12:403-7.

20. Antonia J, Villegas A, Daniel D, Vincente D, Murakami S, Hui R, et al. Durvalumab after chemoradiotherapy in stage III non-small cell lung cancer. $N$ Engl J Med. 2017;377:1919-29.

21. Hellman MD, Chaft JE, William WN Jr, Rusch V, Pisters KMW, Kalhor N, et al. Pathological response after neoadjuvant chemotherapy in resectable non-small cell lung cancers: proposals for the use of major pathological response as a surrogate endpoint. Lancet Oncol. 2014;15:e42-50.

22. Chaft JE, Forde PM, Smith KN, Anagnostou V, Cottress T, Taube JM, et al. Neoadjuvant nivolumab in early stage, resectable non-small cell lung cancers. J Clin Oncol. 2017;35(suppl 15):8508.

23. O'Kane GM, Labbé C, Doherty MK, Young K, Albaba H, Leighl NB. Monitoring and management of immune-related adverse events associated with programmed cell death protein-1 axis inhibitors in lung cancer. Oncologist. 2017;22:70-80

24. Abdel-Rahman O, Fouad M. Risk of pneumonitis in cancer patients treated with immune checkpoint inhibitors: a meta-analysis. Ther Adv Respir Dis. 2016;10: 183-93.

25. Nishino M, Giobbie-Hurder A, Hatabu H, Ramaiya NH, Hodi FS. Incidence of programmed cell death 1 inhibitor-related pneumonitis in patients with advanced cancer. A systematic review and meta-analysis. JAMA Oncol. 2016;2:1607-16. 